EPJ Web of Conferences 52, 09008 (2013)

DOI: $10.1051 /$ epjconf/20135209008

(C) Owned by the authors, published by EDP Sciences, 2013

\title{
The ANTARES neutrino telescope
}

\author{
Annarita Margiotta ${ }^{1,2, a}$ on behalf of the ANTARES Collaboration \\ ${ }^{1}$ Dipartimento di Fisica dell'Università, viale C. Berti Pichat, 6/2, 40127 Bologna, Italy \\ ${ }^{2}$ Sezione INFN - Bologna, viale C. Berti Pichat, 6/2, 40127 Bologna, Italy
}

\begin{abstract}
The ANTARES neutrino telescope is the largest operating undersea neutrino telescope. It is located in the Northern hemisphere, $40 \mathrm{~km}$ far from Toulon, in the Mediterranean Sea. The detector array consists of 12 detector lines holding a total of 885 Optical Modules (OMs) and is located at a depth of about $2500 \mathrm{~m}$. Its main scientific goal is the detection of high energy cosmic neutrinos through measurement of Cherenkov radiation from neutrino-induced charged leptons. Since the completion of the detector in 2008, analysis of the ANTARES data has been continuously improving. Further investigation covers other high energy researches and Earth and sea science studies. The various prospects and current status of the analysis are presented.
\end{abstract}

\section{Introduction}

ANTARES is the largest undersea neutrino telescope. It is located $2500 \mathrm{~m}$ deep in the Mediterranean Sea offshore the French coast, $40 \mathrm{~km}$ far from Toulon. It consists of a 3D lattice of optical sensors (photomultipliers - PMTs) distributed along 12 flexible lines anchored on the sea bed and kept tight with buoys. 25 storeys, each carrying a triplet of PMTs, are typically mounted on each line. The main scientific objective is the search for high energy neutrinos coming from possible Galactic and extra Galactic sources. The detection principle is based on the collection of Cherenkov photons emitted along the path of ultrarelativistic charged particles produced in the neutrino interactions with matter (water or rock) surrounding the detector. Information on charge amplitudes and arrival times of the PMT signals are used by dedicated algorithms in order to reconstruct the direction of the charged particles. A detailed description of the ANTARES detector can be found here [1].

Some aspects that are typical to an undersea neutrino detector and other analyses that have been carrying on, using data collected since 2006, are described in this report. A summary of the most recent results on point source searches, neutrino diffuse flux studies and neutrino oscillation analyses is included in the contribution of J. Brunner at this symposium [2].

\section{Positioning and calibration of the detector}

\subsection{Positioning}

The 12 ANTARES lines are flexible and can float under the continuous action of sea currents. In order to reconstruct muon direction with high precision (better than a

\footnotetext{
ae-mail: margiotta@bo.infn.it
}

few tenths of a degree) good timing resolution and accuracy of the location of the PMTs (better than $10 \mathrm{~cm}$, corresponding to about $0.5 \mathrm{~ns}$ time uncertainty ) are essential. 5 hydrophones (receivers) are distributed along each lines and are part of a high-frequency long-baseline acoustic positioning system, together with some emitters on the sea floor around the detector and at the anchor of each line. In addition, a compass-tiltmeter system is installed for the measurement of the orientation and inclination of the storeys.

Every 2 minutes the positions of the hydrophones are measured with triangulation methods. Starting from that, the shape of each line is reconstructed by a global fit based on a model of the physical properties of the line and taking into account information provided by the tiltmeters and compass sensors located on each storey.

Figure 1 shows the difference between triangulation result and line fit for the position of storey 20 of line 3 in March 2010. Figure 2 shows fitted current velocities from the positioning procedure (averaged over all twelve lines) compared to the ones measured with an Acoustic Doppler Current Profiler (ADCP)located on the ANTARES detector, for a period of one week. More details on the positioning system of the ANTARES detector can be found in [3].

\subsection{Calibration with ${ }^{40} \mathrm{~K}$}

${ }^{40} \mathrm{~K}$ present in sea water is not only background but also an important calibration tool. The decay of ${ }^{40} K$ yields an electron with energy exceeding the Cherenkov threshold for electrons in water $(0.26 \mathrm{MeV})$, and is sufficient to produce up to 150 Cherenkov photons. The measurement of optical rate is used to get a high precision monitoring of the relative efficiencies between OMs.

If the decay occurs close to a detector storey, coincident signals may be recorded by pairs of PMTs on the storey. 


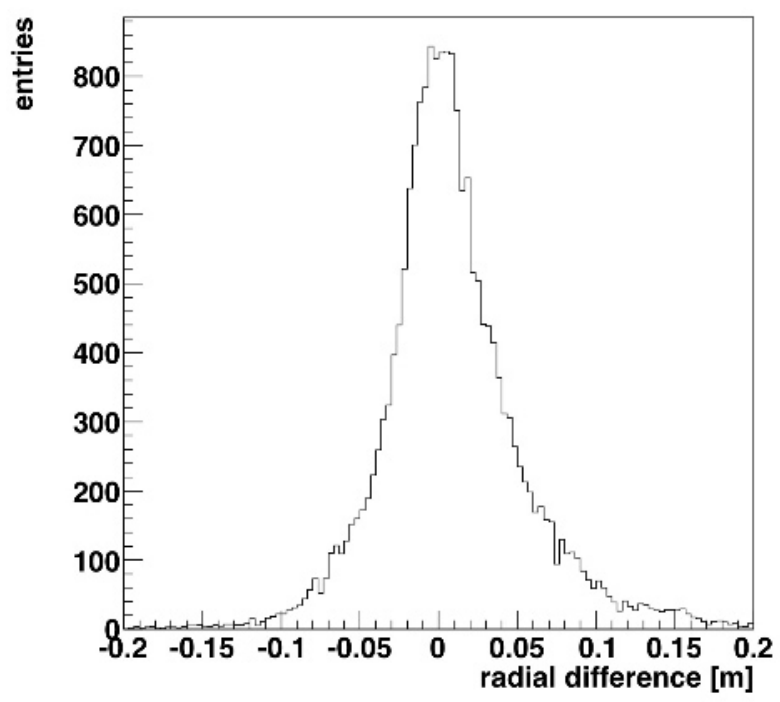

Figure 1. Difference between triangulation result and line fit for the position of storey 20 of Line 3 in March 2010.
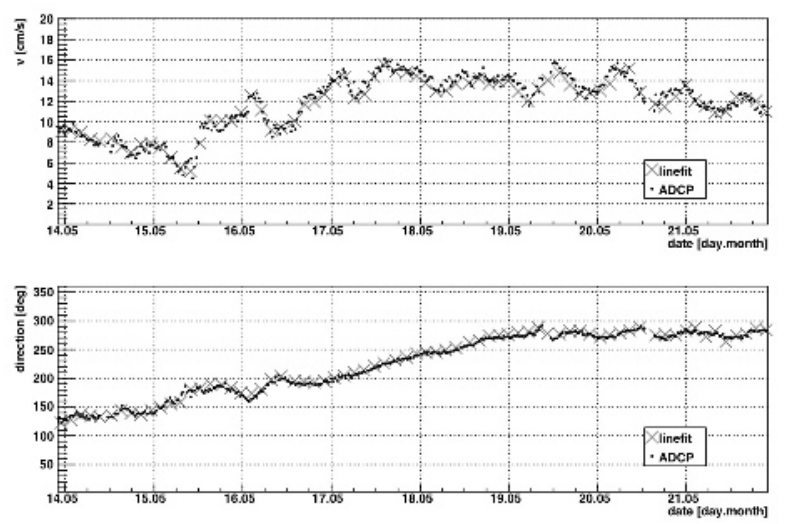

Figure 2. Fitted current velocities from the positioning procedure (averaged over all twelve lines) compared to the ones measured with the ADCP for a period of one week; top: speed of the sea current, bottom: direction of the sea current.

Fig. 3 shows an example of the distribution of the measured time differences between hits in two OMs of the same storey. A peak is present, in good agreement with the expectations from simulations. The data have been fitted to the sum of a Gaussian distribution and a flat background. The width of these distributions (FWHM = $9 \mathrm{~ns}$ ) is set by the difference in the distance from the point where the decay occurs to each of the OMs of the pair. The position of the peak can be used to cross-check the time offsets within each storey provided by the onshore dark room and optical beacon calibrations. Details on the ANTARES calibration procedures can be found in [4].

\section{Atmospheric muons}

Though the main goal of a neutrino telescope is the detection of extraterrestrial neutrinos, the signal in ANTARES

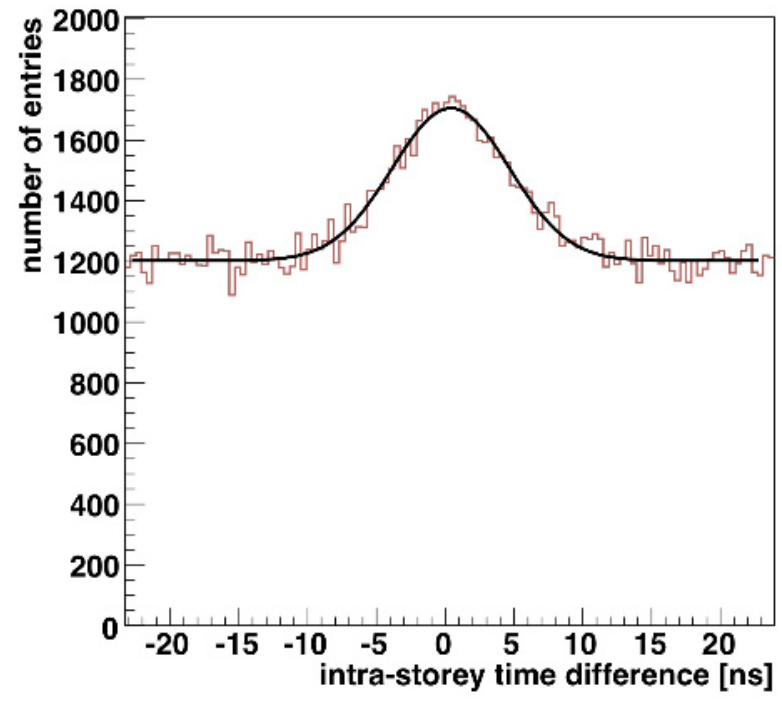

Figure 3. Distribution of background hit time differences for one pair of OMs in the same storey. The peak is due to single ${ }^{40} \mathrm{~K}$ decays detected in coincidence by two OMs.

is mainly due to downgoing muons produced in cosmic ray $(\mathrm{CR})$ interactions with atmospheric nuclei. Measuring the underwater muon flux is an important tool to test reconstruction algorithms and understand the systematics of the detector. Two different studies of the vertical depthintensity relation (DIR) have been performed.

In the first one, the attenuation of the muon flux as a function of water depth is observed as a reduction of coincidence rate between adjacent storeys along the detection lines [5]. In this case no reconstruction algorithm is required and a direct check of the detector response is possible. The result of this method is illustrated in Fig. 4.

The second method to measure the DIR makes use of a reconstruction algorithm [6]. The reconstructed zenith angle is converted into an equivalent water slant depth and, starting from the muon angular distribution, a depth-intensity relation can be extracted [7]. The measurement has been performed using data from the partial 5-line configuration and from the full 12-line detector. The results are in agreement with previous measurements and with Monte Carlo expectations, as shown in Fig. 5. The large error band is mainly due to the uncertainty on the efficiency of the OMs at large angles that affects in particular down-going events and on optical properties of water.

\section{Magnetic Monopoles}

Magnetic monopoles (MM) are foreseen in most Grand Unification Theories as particles produced in the early Universe. Predictions for their mass range from $10^{4}$ to $10^{20} \mathrm{GeV}$ depending on the model. Also their speed can vary over a large range. Measurements and estimates of cosmic magnetic fields suggest that MM lighter than $10^{14} \mathrm{GeV}$ could have been accelerated to relativistic velocities and can produce Cherenkov photons, as electrically charged particles do, [18]. Using a simple Earth model, 


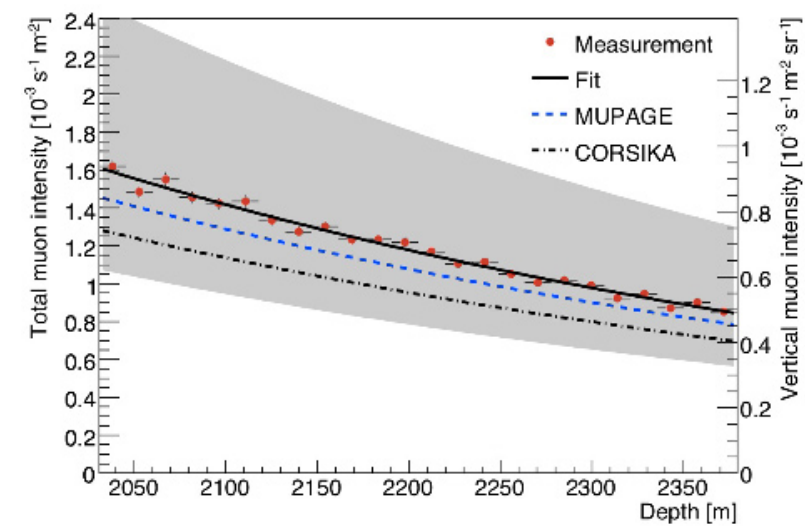

Figure 4. The measured flux of muons as a function of depth. The results can be interpreted in terms of total muon intensity (left axis) as well as vertical muon intensity (right axis). The grey band shows the normalization uncertainty of the data. The predictions of the Monte Carlo simulations, based on different software packages, are shown with dashed (MUPAGE [8]) and dash-dotted (CORSIKA [9]) lines.

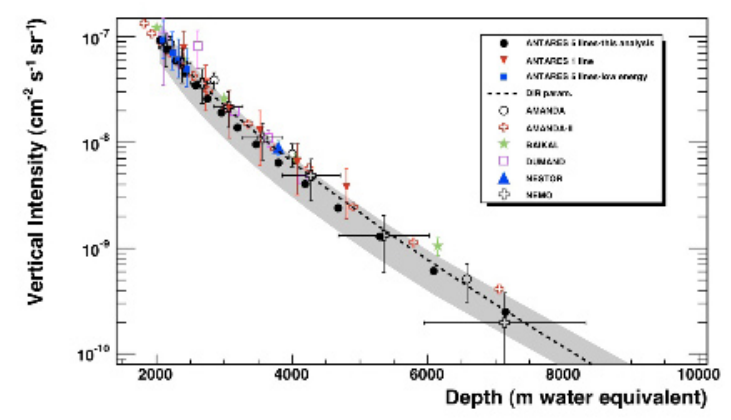

Figure 5. Vertical muon flux of atmospheric muons for the 5 line ANTARES data (black points) as a function of the slant depth. Downward triangles show the results obtained using data collected with the first ANTARES line, [10]. Full squares show the results obtained measuring the muon flux attenuation using the first method described in the text, [5]. Expectation from the Bugaev parametrization (dotted line) is superimposed [11]. A compilation of results obtained with other underwater detectors is shown: AMANDA [12], AMANDA-II [13] Baikal [14], DUMAND [15], NESTOR [16], NEMO [17].

calculations suggest that MM with mass below $10^{14} \mathrm{GeV}$ can be detected in a neutrino telescope after crossing the Earth [19], [20].

A fast MM $\left(\beta=v_{M M} / c>\beta_{\text {threshold }}=0.74\right)$ with $g=g_{D}$, where $g$ is the magnetic charge of the monopole and $g_{D}=\frac{\hbar c}{2 e}$ is the minimal magnetic charge, is expected to produce about 9000 times more Cherenkov photons than a relativistic muon. In addition, a MM with $\beta>0.51$ ionizes sea water leading to indirect Cherenkov emission from $\delta$ rays produced along its path. A search for upgoing relativistic MM has been performed using one year data from the ANTARES detector. No candidate events have been found and upper limits have been put, fig. 6 .

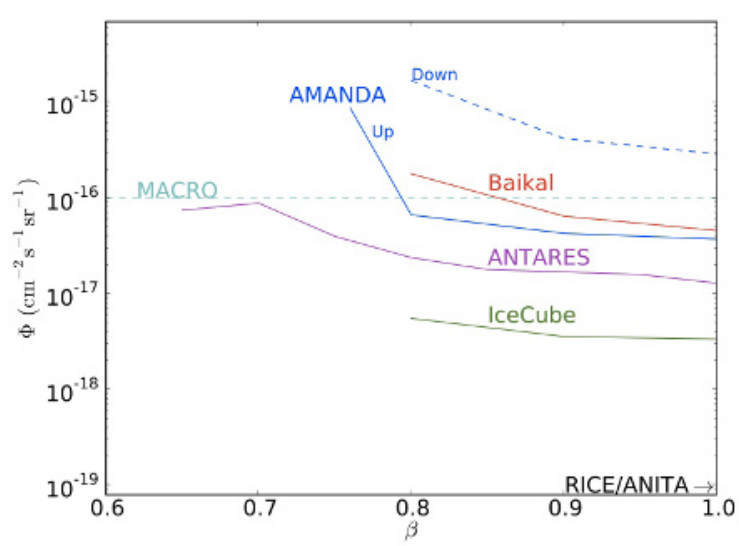

Figure 6. Flux limits (90\% C.L.) on upgoing magnetic monopole flux as a function of $\beta$. Results from AMANDA [21] (also the limit on downgoing MM flux is shown), Baikal [22], MACRO [23] (the limit refers to an isotropic flux of monopoles), IceCube [24], RICE [25] and ANITA [26] are included. The picture is extracted from [24].

\section{Nuclearites}

Nuclearites may have formed in the Early Universe, in a first order quark-hadron phase transition, or as debris of SuperNovae and strange star collisions. They are supposed to be stable, massive lumps of up, down and strange quarks in nearly equal numbers. The existence of strange quark matter nuggets would imply a new ground state of nuclear matter and may explain the dark matter problem. Nuclearites would have velocities of about $10^{-3} c$, typical of objects gravitationally trapped in our Galaxy. Nuclearites moving through water would produce a thermal shock emitting blackbody radiation. Nuclearites with masses $>10^{13} \mathrm{GeV}$ passing through the detector would produce a larger light signal compared to atmospheric muons. For instance, a nuclearite of $10^{15} \mathrm{GeV}$ mass would emit isotropically about $10^{6}$ visible photons $/ \mathrm{cm}$ at the ANTARES depth, crossing the ANTARES detector in a characteristic time of $\sim 1 \mathrm{~ms}$, while muons with $\beta \sim 1$ emit about $4 \cdot 10^{2}$ Cherenkov photons $/ \mathrm{cm}$, in $\sim 2.2 \mu$ s crossing time. A set of specific cuts were defined to identify possible candidates in data collected during 310 days of ANTARES acquisition, in 2007 and 2008. No events were found. In Fig. 7 the established upper limits are shown.

\section{Acoustic measurements}

The ANTARES telescope hosts a prototype system for acoustic detection of neutrinos in the deep sea, AMADEUS (ANTARES Modules for the Acoustic Detection Under the Sea), [27]. The acoustic detection principle is based on the measurement of acoustic pressure pulses produced by particle cascades that evolve when high energy neutrinos $(\mathrm{E}>100 \mathrm{PeV})$ interact with nuclei in water. Completed in May 2008, AMADEUS consists of six "acoustic clusters", installed at water depths between 2050 


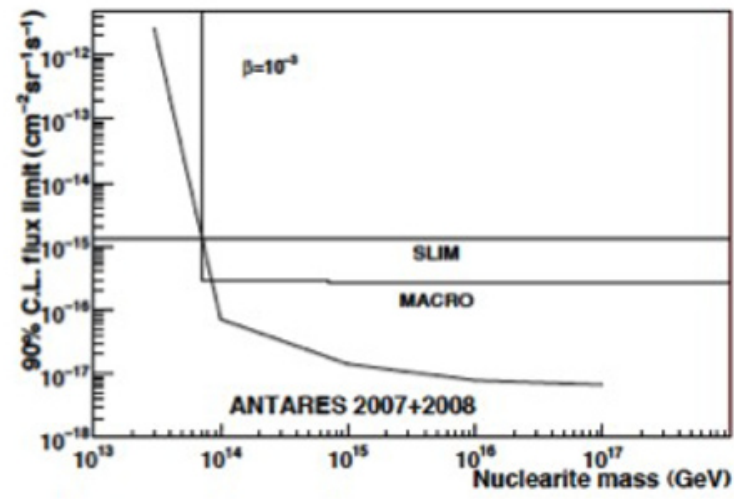

Figure 7. ANTARES sensitivity to nuclearites with the 5, 9, 10 and 12 line combined configurations from 2007-2008, for a total live time of 310.6 days.

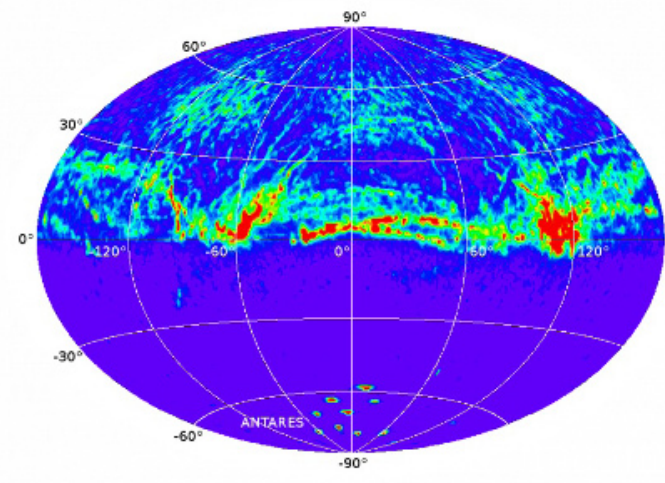

Figure 8. Angular distribution of marine sound sources.

and $2300 \mathrm{~m}$, each comprising six acoustic sensors that employ piezo-electric elements for the broad-band recording of signals with frequencies ranging up to $125 \mathrm{kHz}$ and arranged at distances of roughly $1 \mathrm{~m}$ from each other. Three of them are installed on one of the telescope lines. The others are mounted on a 13th additional line, the so called Instrumentation Line, which carries sea and Earth science devices. Spacings between the clusters range from 14.5 to $340 \mathrm{~m}$. Each cluster contains custom-designed electronics boards to amplify and digitise the acoustic signals from the sensors. An on-shore computer cluster is used to process and filter the data stream and store the selected events. AMADEUS allows extensive studies of both transient signals and ambient noise in the deep sea, as well as signal correlations on several length scales and localisation of acoustic point sources. Fig. 8 shows the space distribution of triggered signals due to ambient noise, represented on a "sky map". In the plot, "hot spots" due to the acoustic positioning system of ANTARES and to major ports (shipping traffic) are clearly visible. This picture shows the capability of AMADEUS to reconstruct the direction of acoustic sources, which is considered a prerequisite to reconstruct neutrino-like signals.

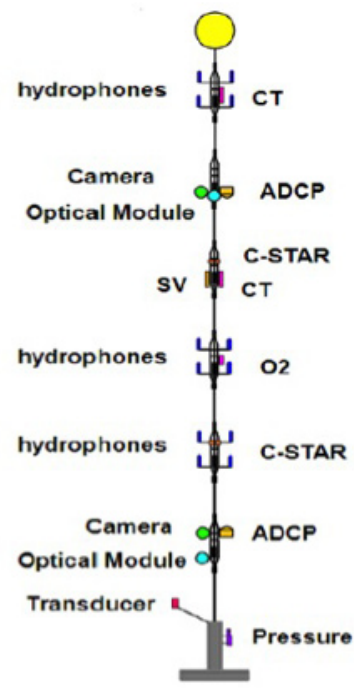

Figure 9. The instrumentation line IL07. Elements are indicated schematically; not drawn to scale.

\section{ANTARES as deep-sea multidisciplinary observatory}

Water surrounding ANTARES acts as shield, target and propagation medium for particles and light. A detailed and accurate monitoring of its properties and of the biological and physical processes happening around the detector is essential to define the input parameters to MonteCarlo simulations and to understand which phenomena can interfere with neutrino telescope operations.

Several Earth and sea science devices are mounted along the lines and in proximity of the telescope, on a 13th line, called the Instrumented Line (IL07). This makes ANTARES a multidisciplinary deep ocean cabled observatory and provides marine scientists with new opportunities for interdisciplinary studies of complex earth-ocean systems.

A sketch of the line and of the hosted devices is in Fig. 9. Several studies have been carrying on in oceanography [28], biology and seismology.

Measurements of salinity, temperature and sea currents can give signals for deep water formation in one of the most active regions for this phenomenon.

Two bio-cameras are installed on the IL07 at 2200 and $2400 \mathrm{~m}$ under sea level. They have been permanently active at the ANTARES site since early 2008 until Nov 2010, when the IL07 was disconnected. Fig. 10 shows some examples of images taken with different exposure times: on the left, a 2-seconds exposure image; in the middle, the sequence of two images with 0.2-seconds exposure; on the right, short flashes visible on one or two single images with $100 \mathrm{~ms}$ exposure. The first two examples are most likely due to bioluminescent organisms. The last image is similar to structures that are visible in standard astrophotography slides, due to traces from charged particle remnants from cosmic ray showers. Registered triggers have been separated in two samples: "cosmic ray triggers", 


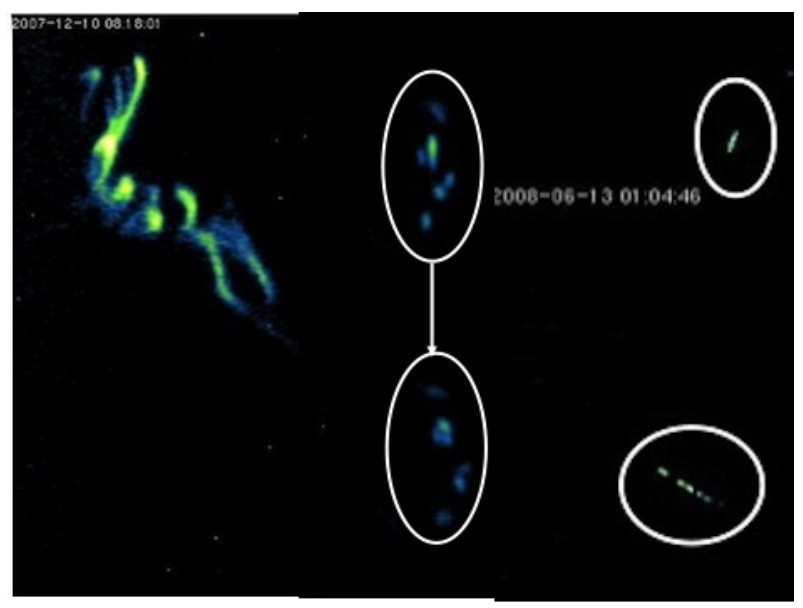

Figure 10. Images taken with bio camera at $2200 \mathrm{~m}$ under s.1., see text for details.

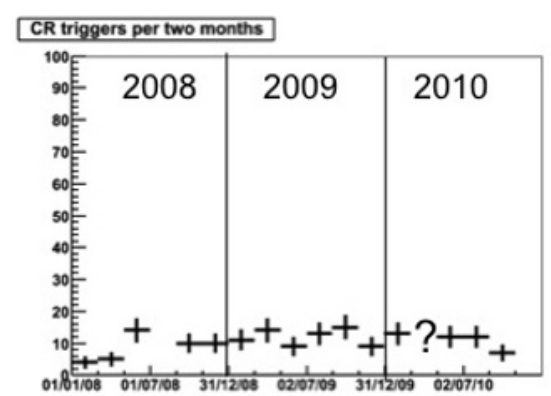

Figure 11. Time distribution of "cosmic ray triggers".

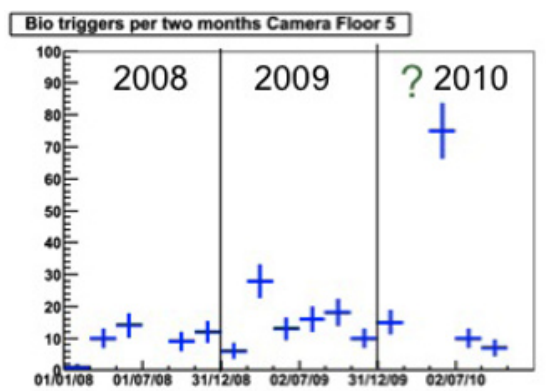

Figure 12. Time distribution of "biological triggers".

Fig. 11, and "biological triggers", Fig. 12. A preliminary quantitative analysis showed that while triggers of biological origin show seasonal and year variations, triggers attributed to CR have essentially a constant frequency, about 5.4 events/month on a surface of $4 \times 4 \mathrm{~cm}^{2}$. This matches with the low energy muon flux evaluated at $2200 \mathrm{~m}$ under s.1. in [5] and shown in Fig. 4. A complete analysis of data collected with bio-cameras is in progress.

\section{Conclusions}

A short survey of analyses and activities that can be performed with an undersea neutrino telescope is given.
ANTARES has proved that the Cherenkov detection technique is feasible even in deep sea water, in neutrino and high energy physics. It has also put in evidence the mutual benefits that can be obtained with the common usage of deep sea infrastructures by the high energy physics and sea science communities.

\section{References}

[1] M. Ageron et al., Nucl. Instrum. Meth. A 656, 11 (2011).

[2] J. Brunner, IceCube and ANTARES, these proceedings.

[3] S. Adrian-Martinez et al., JINST 7, T08002 (2012).

[4] J.A. Aguilar et al., Astropart. Phys. 34, 539 (2011).

[5] J.A. Aguilar et al., Astropart. Phys. 33, 86 (2010); (erratum in Astropart. Phys. 34185 (2010))

[6] J.A. Aguilar et al., Astropart. Phys. 34 (2011) 652.

[7] J.A. Aguilar et al., Astropart. Phys. 34, 179 (2010).

[8] Y. Becherini et al., Astropart. Phys. 25 (2006)1; G. Carminati et al., Comp. Phys. Commun. 179 (2008) 915.

[9] D. Heck et al., Report FZKA 6019, 1998, Forschungszentrum Karlsruhe; D. Heck, J. Knapp, Report FZKA 6097, 1998, Forschungszentrum Karlsruhe.

[10] M. Ageron et al., Astropart. Phys. 31, 277 (2009).

[11] E. V. Bugaev et al., Phys. Rev. D 58, 05401 (1998).

[12] E. Andres et al., Astropart.Phys. 13, 1 (2000).

[13] P. Desiati for AMANDA Collab., Proc. of the 28th ICRC, Tsukuba 2003.

[14] I. A. Belolaptikov et al., Astropart. Phys. 7, 263 (1997).

[15] J. Babson et al., Phys. Rev. D 42, 41 (1990).

[16] G. Aggouras et al., Astropart. Phys. 23, 377 (2005).

[17] S. Aiello et al., Astropart. Phys. 33, 263 (2010).

[18] D.R. Tompkins, Phys. Rev. 138, 248 (1965).

[19] S. Balestra et al, Eur. Phys. J. C 55, 206 (2008).

[20] J. Derkaoui et al., Astropart. Phys. 9, 173 (1998).

[21] R. Abbasi et al., IceCube Coll., Eur. Phys. J. C 69, 361 (2010).

[22] V. Aynutdinov et al., BAIKAL Coll., Astropart. Phys. 29, 366 (2008).

[23] M. Ambrosio et al., MACRO Coll., Eur. Phys. J. C 25, 511 (2002).

[24] R. Abbasi et al., IceCube Coll., Phys. Rev. D 87, 022001 (2013).

[25] D.P. Hogan, D.Z. Besson, J.P. Ralston, I. Kravchenko, and D. Seckel, Phys. Rev. D 78075031 (2008).

[26] M. Detrixhe et al. (ANITA Collaboration), Phys. Rev. D 83023513 (2011).

[27] J.A. Aguilar et al., Nucl. Instrum. Meth. A 626-627, 128 (2011).

[28] H. van Haren et al., Deep-Sea Research I 58, 875 (2011). 